\title{
Searching for molecular hydrogen mid-infrared emission in the circumstellar environments of Herbig Be stars (Research Note)
}

\author{
C. Martin-Zaïdi ${ }^{1}$, E. F. van Dishoeck ${ }^{2,3}$, J.-C. Augereau ${ }^{1}$, P.-O. Lagage ${ }^{4}$, and E. Pantin ${ }^{4}$ \\ ${ }^{1}$ Laboratoire d'Astrophysique de Grenoble, CNRS, Université Joseph-Fourier, UMR 5571, 38041 Grenoble Cedex 09, France \\ e-mail: claire.martin-zaidi@obs.ujf-grenoble.fr \\ 2 Leiden Observatory, Leiden University, PO Box 9513, 2300 RA Leiden, The Netherlands \\ Max Planck Institut für Extraterrestrische Physik, Giessenbachstrasse 1, 85748 Garching, Germany \\ ${ }^{4}$ Laboratoire AIM, CEA/DSM, CNRS, Université Paris Diderot, DAPNIA/Service d'Astrophysique, Bât. 709, CEA/Saclay, \\ 91191 Gif-sur-Yvette Cedex, France
}

Received 14 February 2008 / Accepted 30 June 2008

\begin{abstract}
Context. Molecular hydrogen $\left(\mathrm{H}_{2}\right)$ is the most abundant molecule in the circumstellar (CS) environments of young stars, and is a key element in giant planet formation. The measurement of the $\mathrm{H}_{2}$ content provides the most direct probe of the total amount of CS gas, especially in the inner warm planet-forming regions of the disks.

Aims. Most Herbig Be stars (HBes) are distant from the Sun and their nature and evolution are still debated. We therefore conducted mid-infrared observations of $\mathrm{H}_{2}$ as a tracer of warm gas around HBes known to have gas-rich CS environments.

Methods. We report a search for the $\mathrm{H}_{2} \mathrm{~S}(1)$ emission line at $17.0348 \mu \mathrm{m}$ in the CS environments of $5 \mathrm{HBes}$ with the high resolution spectroscopic mode of VISIR (ESO VLT Imager and Spectrometer for the mid-InfraRed).

Results. No source shows evidence for $\mathrm{H}_{2}$ emission at $17.0348 \mu \mathrm{m}$. Stringent $3 \sigma$ upper limits on the integrated line fluxes are derived. Depending on the adopted temperature, limits on column densities and masses of warm gas are also estimated. These non-detections constrain the amount of warm $(>150 \mathrm{~K})$ gas in the immediate CS environments of our target stars to be less than $\sim 1-10 M_{\text {Jup }}$.
\end{abstract}

Key words. stars: circumstellar matter - stars: formation - stars: pre-main sequence - ISM: molecules

\section{Introduction}

Molecular hydrogen $\left(\mathrm{H}_{2}\right)$ is the most abundant molecule in the environment of young stars. It remains optically thin up to high column densities $\left(\sim 10^{23} \mathrm{~cm}^{2}\right)$, and does not freeze onto dust grain surfaces. It furthermore self-shields efficiently against photodissociation by far-ultraviolet (FUV) photons. Therefore, $\mathrm{H}_{2}$ is the only molecule that can directly constrain the mass reservoir of molecular gas in the circumstellar (CS) environment of pre-main sequence stars. On the other hand, $\mathrm{H}_{2}$ is one of the most challenging molecules to detect. Electronic transitions occur in the UV to which the Earth's atmosphere is opaque, and rotational and rovibrational transitions at infrared (IR) wavelengths are faint because of their quadrupolar origin. FUV spectroscopic observations of $\mathrm{H}_{2}$ absorption lines have provided evidence for the presence of cold and warm excited $\mathrm{H}_{2}$ around numerous Herbig Ae/Be stars (HAeBes) but have not allowed us to determine the spatial distribution of the observed gas (e.g. Martin-Zaïdi et al. 2008). Detections of $\mathrm{H}_{2}$ line emission from disks around young stars with ISO (Thi et al. 2001) were contradicted by ground-based observations (Richter et al. 2002; Sako et al. 2005), which showed that the observed emission could be due to the surrounding cloud material. Carmona et al. (2008) modeled the mid-IR $\mathrm{H}_{2}$ lines originating in a gas-rich disk, seen face-on, surrounding a Herbig Ae (HAe) star at $140 \mathrm{pc}$ from the Sun. By assuming that the gas and dust were well-mixed in the disk, a gas-to-dust ratio of about 100 , and that $T_{\text {gas }}=T_{\text {dust }}$, those authors demonstrated that mid-IR $\mathrm{H}_{2}$ lines could not be detected with the existing instruments. Indeed, they were unable to detect any $\mathrm{H}_{2}$ mid-IR emission line in their sample of 6 HAe stars. At the present time, observations of $\mathrm{H}_{2}$ mid-IR emission lines have been reported in 8 of 76 T Tauri stars observed with Spitzer (Lahuis et al. 2007). Although mid-IR windows are strongly affected by sky and instrument background emission, the advent of high spectral and spatial resolution spectrographs allows us to study the $\mathrm{H}_{2}$ emission from the ground, as demonstrated in the cases of two HAeBes, HD 97048 (Martin-Zaïdi et al. 2007) and AB Aur (Bitner et al. 2007), for which particular conditions are required in their CS disks. The analyses of these data usually assumes that the $\mathrm{H}_{2}$ excitation is in local thermodynamic equilibrium (LTE) and can thus be characterized by a single excitation temperature, which should be close to the gas temperature because of the low critical densities. The AB Aur observations infer an $\mathrm{H}_{2}$ gas temperature that is significantly higher than the dust temperature.

In contrast to HAes, the more distant Herbig Be stars (HBes) earlier than B9 type have been poorly studied. As a consequence, the nature and evolution of the CS material surrounding HBes is still a subject of controversy. The Spectral Energy Distributions (SEDs) are very different from one $\mathrm{HBe}$ to another (e.g. Hillenbrand et al. 1992). Some have SEDs that are comparable to those observed for HAes, and have been classified as group I or group II stars haboring disks according to the classification of Meeus et al. (2001). However, numerous HBes SEDs with little IR excess have been modeled successfully by 
Table 1. Astrophysical parameters of the sample stars (Cols. 2 to 6). $v_{\text {rad }}$ is the radial velocity of the star in the heliocentric rest frame. Columns 7 to 12 summarize the observations. The airmass and seeing intervals are given from the beginning to the end of the observations.

\begin{tabular}{|c|c|c|c|c|c|c|c|c|c|c|c|}
\hline Star & $\begin{array}{l}\text { Sp. } \\
\text { Type }\end{array}$ & $\begin{array}{l}T_{\text {eff }} \\
(\mathrm{K})\end{array}$ & $\begin{array}{c}A_{\mathrm{v}} \\
\text { (mag) }\end{array}$ & $\begin{array}{c}v_{\mathrm{rad}} \\
\left(\mathrm{km} \mathrm{s}^{-1}\right)\end{array}$ & $\begin{array}{c}d \\
(\mathrm{pc})\end{array}$ & $\begin{array}{l}t_{\text {exp }} \\
(\mathrm{s})\end{array}$ & Airmass & $\begin{array}{c}\text { Optical } \\
\text { Seeing } \\
\left({ }^{\prime \prime}\right)\end{array}$ & $\begin{array}{c}\text { Standard } \\
\text { Star }\end{array}$ & Airmass & $\begin{array}{c}\text { Optical } \\
\text { Seeing } \\
\left({ }^{\prime \prime}\right)\end{array}$ \\
\hline HD 98922 & B9 & $10470^{(1)}$ & $0.34^{(1)}$ & $-15^{(2)}$ & $>540^{(1)}$ & 2700 & $1.14-1.15$ & $0.76-1.41$ & HD 25025 & $1.024-1.025$ & $0.69-0.71$ \\
\hline HD 250550 & B7 & $12800^{(3)}$ & $0.57^{(3)}$ & $+31^{(4)}$ & $606 \pm 367^{(5)}$ & 3600 & $1.32-1.50$ & $0.83-1.50$ & HD 93813 & $1.018-1.023$ & $0.91-1.05$ \\
\hline HD 259431 & B5 & $15900^{(3)}$ & $0.88^{(3)}$ & $+43^{(4)}$ & $290 \pm 84^{(5)}$ & 2250 & $1.23-1.32$ & $0.75-1.28$ & HD 39425 & $1.12-1.14$ & $1.51-1.62$ \\
\hline HD 76534 & B2 & $20000^{(6)}$ & $0.80^{(7)}$ & $+17^{(4)}$ & $>160^{(1)}$ & 3600 & $1.05-1.14$ & $0.85-1.25$ & HD 93813 & $1.014-1.017$ & $0.92-0.96$ \\
\hline HD 45677 & $\mathrm{~B} 2 / \mathrm{B} 1$ & $21400^{(1)}$ & $0.87^{(1)}$ & $+21.6^{(8)}$ & $500^{(9)}$ & 3450 & $1.02-1.08$ & $0.69-1.40$ & HD 25025 & $1.024-1.025$ & $0.69-0.71$ \\
\hline
\end{tabular}

References: (1) van den Ancker et al. (1998); ${ }^{(2)}$ Acke et al. (2005); ${ }^{(3)}$ Bouret et al. (2003); ${ }^{(4)}$ Finkenzeller \& Jankovics (1984); ${ }^{(5)}$ Brittain et al. (2007); ${ }^{(6)}$ Martin et al. (2004); (7) Valenti et al. (2000); ${ }^{(8)}$ Evans (1967); ${ }^{(9)}$ Zorec et al. (1998) as quoted in Cidale et al. (2001).

free-free emission originating in a gaseous CS envelope, and compared with rapidly rotating classical Be stars. In addition, the mid-IR emission observed in HBes is generally not confined to optically thick disks but originates in more complex environments such as remnant envelopes or halos (Leinert et al. 2001; Polomski et al. 2002; Vinković et al. 2006). This implies that there are structural differences between HAes and the more luminous HBes (Natta et al. 2000; Leinert et al. 2001). These results are fully consistent with the more rapid evolution of the more massive HAeBes, which should still be partially embedded in their natal cloud. On the other hand, near-IR interferometric data of HBes have been interpreted using flared disks models (e.g. Kraus et al. 2007).

We selected five HBe stars whose CS environments are rich in gas as observed by the FUSE satellite (Martin-Zaïdi et al. 2008). The selected stars have IR excesses that are assumed to represent the presence of CS dusty disks (e.g. Hillenbrand et al. 1992; Polomski et al. 2002), but the disk scenario has not yet been clearly established. The aim of our study is to detect the $\mathrm{H}_{2}$ pure rotational emission line at $17.0348 \mu \mathrm{m}$ in the vicinity of these stars with the high resolution mode of the VLT/VISIR spectrograph (Lagage et al. 2004) and constrain the warm CS gaseous component.

\section{Selection of the target stars}

The selection of the five target stars was motivated by the previous observations of $\mathrm{H}_{2}$ electronic lines in the FUV spectral range, which showed that the $\mathrm{CS}$ environments of HBes were rich in cold and warm $\mathrm{H}_{2}$ (Martin-Zaïdi et al. 2008). The detection of $\mathrm{H}_{2}$ absorption lines provided evidence for the presence of cold $(\sim 100 \mathrm{~K})$ and warm/hot CS gas (up to $1500 \mathrm{~K}$ ) around these stars. We first briefly describe the CS environments of each of the five sample stars. The main astrophysical parameters of the stars are summarized in Table 1.

HD 98922: this star is classified as a self-shadowed disk on the basis its SED (van Boekel et al. 2003). Its optical spectrum displays broad, but relatively weak [O I] emission profiles, which cannot originate in the dusty disk surface but possibly in a rotating gaseous disk inside the dust-sublimation radius (Acke et al. 2005). Our preliminary analysis of its FUSE spectrum indicates that the $\mathrm{CS}$ environment of this star is rich in $\mathrm{H}_{2}$. The excitation of $\mathrm{H}_{2}$ appears to be similar to the HBes observed by Martin-Zaïdi et al. (2008). The modeling of the excitation conditions with the Meudon PDR Code (Le Petit et al. 2006) allows us to conclude that the observed gas is probably located in the remnant cloud where the star formed. We emphasize that no $\mathrm{H}_{2}$
mid-IR line was detected by the Spitzer Space Telescope towards this star (Lahuis et al. 2007).

HD 250550 and HD 259431: the SEDs of these two stars show signs of strong near-IR excesses that were modeled with flat optically thick accretion disks by Hillenbrand et al. (1992). Spectro-polarimetric observations provided indications of flattened structures surrounding the stars (Vink et al. 2002). However, spectroscopic observations provided no evidence for Keplerian rotation with single-peaked emission lines detected for both $\mathrm{HI}$ Br $\gamma$ and CO (Brittain et al. 2007). The excitation of $\mathrm{H}_{2}$ in the CS environments of these stars, revealed by the FUSE satellite, favored an interpretation in terms of CS envelopes which were the remnants of the parent molecular clouds in which the stars were formed (Bouret et al. 2003; Martin-Zaïdi et al. 2008).

HD 76534: Hillenbrand et al. (1992) analyzed the SED of HD 76534 and stressed that the low near-IR excess was probably due to free-free emission in an ionized envelope rather than CS dust. By analyzing FUV absorption lines of $\mathrm{H}_{2}$ and deriving the excitation conditions of the gas around the star, Martin et al. (2004) concluded that the CS environment of HD 76534, which is a B2 star, is probably too hostile for the presence of a CS disk.

HD 45677: the measured infrared excess was attributed to a dust shell surrounding HD 45677 by Swings \& Allen (1971). The presence of a disk was inferred by polarization and UV spectroscopic measurements of accreting gas (Schulte-Ladbeck et al. 1992; Grady et al. 1993); these studies also agreed with the presence of an actively accreting CS disk around the star. The presence of such a disk is confirmed by the analysis of emission lines from ionized metals by Muratorio et al. (2006). Our preliminary analysis of the FUSE spectrum of this star shows the presence of two or more gaseous components along the line of sight. This may be consistent with the presence of a disk associated with a CS envelope.

\section{Observations and data reduction}

Searches for mid-IR $\mathrm{H}_{2}$ lines can be undertaken with the ESO/VISIR instrument due to its high spectral and spatial resolution. Its high spectral resolution $(10000<R<30000)$ allows us to disentangle the $\mathrm{H}_{2}$ lines from the absorption lines due to the Earth's atmosphere. VISIR offers access to the most intense pure rotational lines of $\mathrm{H}_{2}$ : the $\mathrm{S}(1)$ line at $17.0348 \mu \mathrm{m}, \mathrm{S}(2)$ at $12.2786 \mu \mathrm{m}, \mathrm{S}(3)$ at $9.6649 \mu \mathrm{m}$, and $\mathrm{S}(4)$ at $8.0250 \mu \mathrm{m}$. The $\mathrm{S}(0)$ transition close to $28 \mu \mathrm{m}$ is not observable from the ground due to the Earth's atmospheric absorption, and the S(3) line, which is located amidst a forest of telluric ozone features, is only observable for particularly favorable Doppler shifts. 

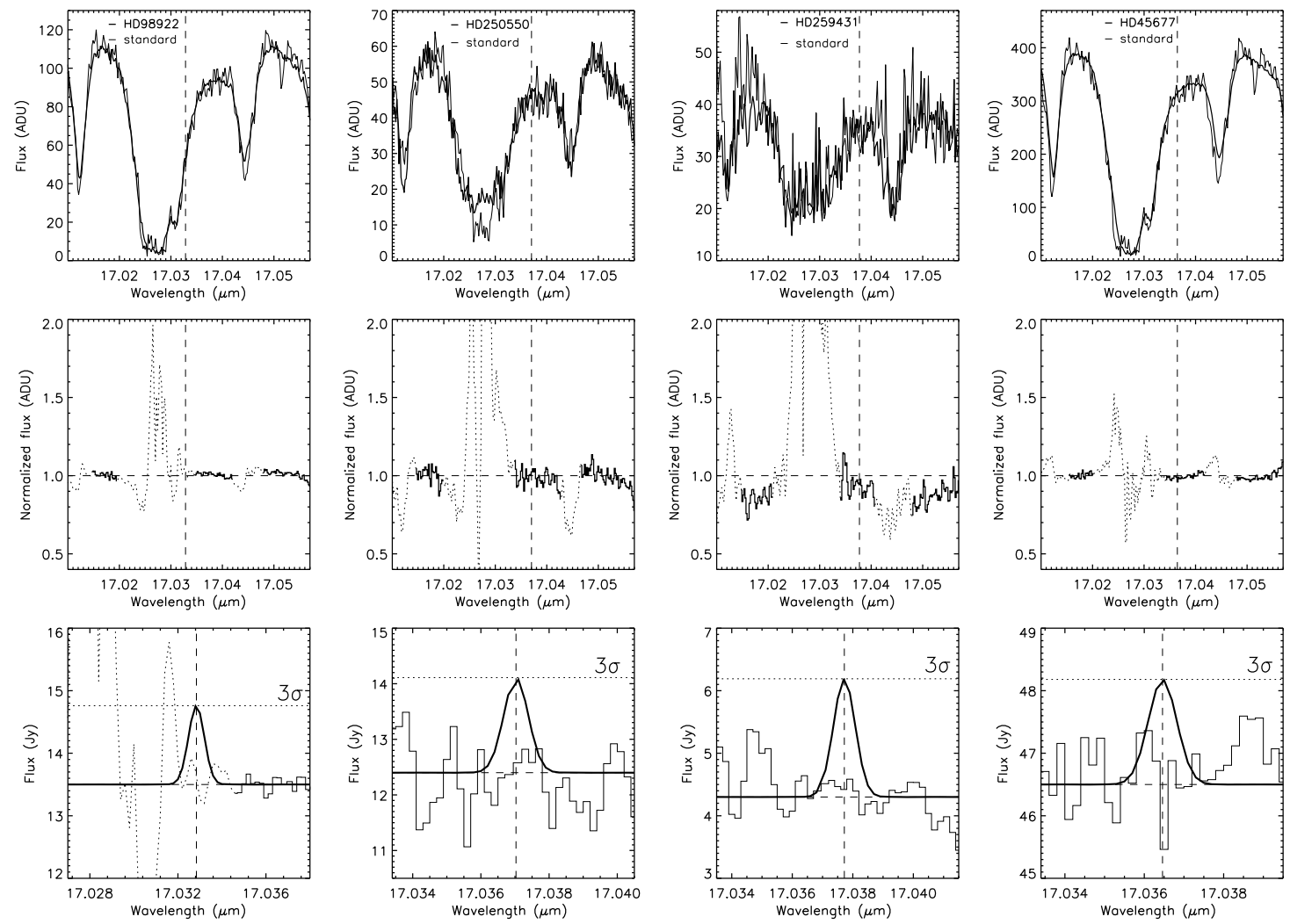

Fig. 1. Spectra obtained for the $\mathrm{H}_{2} \mathrm{~S}(1)$ line at $17.0348 \mu \mathrm{m}$. Top panel: continuum spectra of the standard star and of the target before telluric correction. Central panel: full corrected spectra: dotted lines show spectral regions strongly affected by telluric features. Bottom panel: zoom of the region where the $\mathrm{S}(1)$ line should be observed (dashed vertical lines). A Gaussian of width $F W H M=21 \mathrm{~km} \mathrm{~s}^{-1}$ and integrated line flux equal to the $3 \sigma$ line-flux upper limits is overplotted. The spectra were corrected neither for the radial velocity of the targets nor the Earth's rotation velocity.

The five sample stars were observed with the high spectral resolution long-slit mode of VISIR on January 15 and 16, 2006. The central wavelength of the observations was set to be $17.035 \mu \mathrm{m}$. We used the $0.75^{\prime \prime}$ slit, providing a spectral resolution of about 14000 . The details of the observation conditions are summarized in Table 1. According to its IRAS fluxes, HD 76534 should be observable in the spectral range of VISIR. However, during the observation, the mid-IR flux of this star appeared to be very low and the resulting spectrum could not be used to complete an accurate analysis.

For all other observations, the standard "chopping and nodding" technique was used to suppress the large sky and telescope background dominating at mid-IR wavelengths (for details of the observation technique see Martin-Zaïdi et al. 2007). The chopping was performed with a 8 " amplitude, which enabled us to detect the gas of any elongated structure around our targets. To correct the spectrum for the Earth atmospheric absorption and to obtain the absolute flux calibration, we observed standard stars $^{1}$ immediately before and after observing our sources. Since the HD 45677 and HD 98922 spectra have far higher signalto-noise $(\mathrm{S} / \mathrm{N})$ ratios than that of the standard stars, we divided the spectra of HD 98922, HD 250550, and HD 259431 by that of HD 45677, and divided the spectrum of HD 45677 by that of HD 98922, to correct for the telluric absorption. We checked that the Doppler shifts were sufficiently different from one star to the other, to ensure that the analysis would not be significantly affected. We used the observed and modeled spectra of

\footnotetext{
${ }^{1}$ http://www.eso.org/sci/facilities/paranal/ instruments/visir/tools/
}

the standard stars (Cohen et al. 1999) to derive the absolute flux calibration. The wavelength calibration was completed by fitting the observed sky background features with a model of Paranal's atmospheric emission. The spectra were not corrected for dust extinction, which is negligible in the mid-IR for $A_{\mathrm{v}}<40 \mathrm{mag}$ (Fluks et al. 1994).

\section{Data analysis}

For the four high $\mathrm{S} / \mathrm{N}$ spectra of stars in our sample, we display in Fig. 1, the spectra of the standard stars overplotted on the target spectra (top panel), the entire atmosphere-corrected spectra before the flux calibration (central panel), and the region of the flux-calibrated atmosphere-corrected spectra where the $\mathrm{S}(1) \mathrm{H}_{2}$ line should appear if present (bottom panel). None of the observed sources showed evidence for $\mathrm{H}_{2}$ emission close to $17.035 \mu \mathrm{m}$.

In the flux-calibrated spectra, we used two different methods to determine the standard deviation $(\sigma)$ of the continuum flux. First, we calculated the standard deviation for wavelength ranges relatively unaffected by telluric absorption, and close to the wavelength of interest. Second, we estimated $\sigma$ in regions of the spectra with comparable atmospheric transmission to the line of interest. The first method provided more conservative values of $\sigma$ for all but one star, namely HD 98922; for this star, the line of interest should be detected at wavelengths corresponding to the strong telluric absorption line, and the atmospheric transmission played an important role. For our analysis, we kept the more conservative values, and used the $\sigma$ values obtained with 
Table 2. $3 \sigma$ upper confidence limits on the integrated fluxes and intensities of the $\mathrm{S}(1)$ line, and upper limits on the total column densities of $\mathrm{H}_{2}$ and masses of $\mathrm{H}_{2}$ as a function of the adopted temperature. $\lambda_{\mathrm{obs}}$ is the expected position of the line in the observed spectra.

\begin{tabular}{|c|c|c|c|c|c|c|c|c|c|}
\hline \multirow[t]{2}{*}{$\begin{array}{l}\text { Star } \\
\text { HD }\end{array}$} & \multirow[t]{2}{*}{$\begin{array}{c}\lambda_{\mathrm{obs}} \\
(\mu \mathrm{m})\end{array}$} & \multirow[t]{2}{*}{$\begin{array}{l}\text { Integrated flux } \\
\left(\mathrm{erg} \mathrm{s}^{-1} \mathrm{~cm}^{-2}\right)\end{array}$} & \multirow[t]{2}{*}{$\begin{array}{c}\text { Intensity } \\
\left(\mathrm{erg} \mathrm{cm}^{-2} \mathrm{~s}^{-1} \mathrm{sr}^{-1}\right)\end{array}$} & \multicolumn{3}{|c|}{$\begin{array}{c}N\left(\mathrm{H}_{2}\right) \text { upper limits } \\
\left(\mathrm{cm}^{-2}\right)\end{array}$} & \multicolumn{3}{|c|}{$\begin{array}{l}\mathrm{H}_{2} \text { mass upper limits }{ }^{a} \\
\quad\left(M_{\text {Jup }} \sim 10^{-3} M_{\odot}\right)\end{array}$} \\
\hline & & & & $150 \mathrm{~K}$ & $300 \mathrm{~K}$ & $1000 \mathrm{~K}$ & $150 \mathrm{~K}$ & $300 \mathrm{~K}$ & $1000 \mathrm{~K}$ \\
\hline 98922 & 17.0328 & $.2 \times 10^{-14}$ & $<1.2 \times 10^{-3}$ & $1.1 \times 10^{23}$ & $6.9 \times 10^{21}$ & $1.6 \times 10^{21}$ & 3.3 & $2.0 \times 10^{-1}$ & $5.9 \times 10^{-2}$ \\
\hline 250550 & 17.0373 & $<1.6 \times 10^{-14}$ & $<3.8 \times 10^{-3}$ & $1.5 \times 10^{23}$ & $9.3 \times 10^{21}$ & $2.2 \times 10^{21}$ & $5.5_{-4.7}^{+8.8}$ & $3.4_{-2.9}^{+5.4} \times 10^{-1}$ & $1.0_{-0.8}^{+1.6} \times 10^{-1}$ \\
\hline 259431 & 17.0377 & $<1.8 \times 10^{-14}$ & $<4.2 \times 10^{-3}$ & $1.6 \times 10^{23}$ & $1.0 \times 10^{22}$ & $2.4 \times 10^{21}$ & $1.4_{-0.7}^{+0.9}$ & $8.6_{-4.3}^{+5.7} \times 10^{-2}$ & $2.5_{-1.3}^{+1.7} \times 10^{-2}$ \\
\hline 45677 & 17.0365 & $<1.6 \times 10^{-14}$ & $<3.7 \times 10^{-3}$ & $1.4 \times 10^{23}$ & $9.1 \times 10^{21}$ & $2.1 \times 10^{21}$ & 3.7 & $2.3 \times 10^{-1}$ & $6.7 \times 10^{-2}$ \\
\hline
\end{tabular}

${ }^{a}$ Masses of $\mathrm{H}_{2}$ are calculated assuming the distances quoted in Table 1 (see text). For HD 98922, the lower limit on the distance is used and the mass scales with the distance as $d^{2}$.

the first method for HD 250550, HD 259431, and HD 45677, and the value given by the second method for HD 98922. The $3 \sigma$ upper limits on the integrated line fluxes were calculated by integrating over a Gaussian of full width at half maximum (FWHM) equal to a spectral resolution element $\left(\Delta v \sim 21 \mathrm{~km} \mathrm{~s}^{-1}\right)$, and an amplitude of about $3 \times \sigma \times$ (flux), centered on the expected wavelength for the $S(1)$ line (Fig. 1). The upper limits on integrated fluxes and intensities of the S(1) line derived for each star are tabulated in Table 2. These limits were typically a factor of 2 lower than the detection towards HD 97048 (Martin-Zaïdi et al. 2007). The intensities were calculated by dividing the integrated fluxes by the solid angle of the slit of about $0.75^{\prime \prime}$ width with a spatial resolution element of about $0.427^{\prime \prime}$.

From the upper limits on integrated intensities and by assuming that the emitting $\mathrm{H}_{2}$ is optically thin at LTE, we estimated the total column densities of $\mathrm{H}_{2}$ (for details on the method, see van Dishoeck 1992) as a function of adopted temperatures (150, $300,1000 \mathrm{~K})$. Even though the FUSE data indicated the presence of a gas component with $T \sim 100 \mathrm{~K}$ for our target stars, we measured $150 \mathrm{~K}$ to be the lowest temperature of the gas, which can emit in the $\mathrm{H}_{2} \mathrm{~S}(1)$ line. For temperatures below $150 \mathrm{~K}$, the $\mathrm{S}(0)$ line at $28 \mu \mathrm{m}$ (not observable from the ground) provides more reliable constraints on the optically thin gas properties than the $\mathrm{S}(1)$ line.

Under the same assumptions and assuming a homogeneous medium, the mass of warm $\mathrm{H}_{2}$ is given by:

$$
M_{\text {warm gas }}=f \times 1.76 \times 10^{-20} \frac{F_{u l} d^{2}}{(h c / 4 \pi \lambda) A_{u l} x_{u}(T)} \quad M_{\odot},
$$

where $F_{u l}$ is the line flux, $d$ is the distance in pc to the star, $\lambda$ is the wavelength of the transition $u-l, A_{u l}$ is the spontaneous transition probability, $x_{u}(T)$ is the fractional population of the level $u$ at the temperature $T$ in LTE, and $f$ is the conversion factor required for deriving the total gas mass from the $\mathrm{H}_{2}$-ortho or $\mathrm{H}_{2}$-para mass, assuming that ortho/para ratio is controlled by the gas temperature (from Eq. (1) in Takahashi 2001). Our results are presented in Table 2. The upper limits on the masses are calculated by assuming the distances quoted in Table 1. For HD 98922, we used the lower limit on the distance and its mass scales with the distance as $d^{2}$.

\section{Discussion}

We observed five HBe stars with the high resolution spectroscopic mode of VISIR to search for $\mathrm{H}_{2}$ pure rotational $\mathrm{S}(1)$ emission at $17.0348 \mu \mathrm{m}$. As probed by previous FUSE observations, the CS environments of these stars provide evidence of large reservoirs $\left(N\left(\mathrm{H}_{2}\right) \sim 10^{21} \mathrm{~cm}^{2}\right)$ of cold $\mathrm{H}_{2}(T \sim 100 \mathrm{~K})$, and also evidence for the presence of warm/hot excited $\mathrm{H}_{2}(T \geq 500 \mathrm{~K})$.
The $\mathrm{H}_{2}$, observed by FUSE, probably corresponds to the remnant of the molecular cloud in which the stars were formed and the observed $\mathrm{H}_{2}$ FUV lines do not originate in a CS disk. Martin-Zaïdi et al. (2008) modeled the excitation conditions of $\mathrm{H}_{2}$ and the FUSE spectra of HBes stars using the Meudon PDR Code and showed that the observed gas was located in relatively diffuse regions $\left(10-3000 \mathrm{~cm}^{-3}\right)$ at significant distances from the central stars $(0.03-1.5 \mathrm{pc})$.

None of the four targets with spectra of sufficiently high $\mathrm{S} / \mathrm{N}$ showed any evidence for $\mathrm{H}_{2}$ emission. From the $3 \sigma$ upper limits to the emission line flux, we calculated upper limits on the column density and mass of $\mathrm{H}_{2}$ for each star. We found that the column densities should be lower than $\sim 10^{23} \mathrm{~cm}^{-2}$ at $150 \mathrm{~K}$, and lower than $\sim 10^{21} \mathrm{~cm}^{-2}$ at $1000 \mathrm{~K}$, which does not contradict the column densities derived from the FUSE observations. Upper limits to the masses of warm gas have been estimated to be in the range from $\sim 10^{-2}$ to $\sim 6 M_{\text {Jup }}\left(1 M_{\text {Jup }} \sim 10^{-3} M_{\odot}\right)$, assuming LTE excitation, and depending on the adopted temperature.

It should be pointed out that mid-IR $\mathrm{H}_{2}$ lines only probe warm gas located in the surface layers of the observed media because of the opacity of the interior layers when dust is present. The surface layers of any potential disks surrounding our target stars do not therefore contain sufficient warm gas to enable it to be detected in emission at mid-IR wavelenghs. Following the calculations of Carmona et al. (2008), the $\mathrm{H}_{2}$ mid-IR lines produced by a gas-rich disk with $T_{\text {gas }}=T_{\text {dust }}$ should not be observable with existing instruments. As shown by Bitner et al. (2007) and Martin-Zaïdi et al. (2007), the warm $\mathrm{H}_{2}$ can be detected in the CS disk of a Herbig star, but to explain the detection, particular conditions have to be assumed for the gas and dust, such as $T_{\text {gas }}>T_{\text {dust }}$, which may be created by gas heating by X-rays or UV photons. Our non-detections therefore imply either that the gas and dust in any potential disks are well mixed and have almost equal temperatures, or that these disks are simply not present at all.

Acknowledgements. This work is based on observations obtained at ESO/VLT (Paranal) with VISIR, program number 076.C-0299. C.M.Z. warmly thank M. Deleuil and J.-C. Bouret for their help in preparing the observations. We thank X. Delfosse (LAOG) and the anonymous referee for their fruitful comments about the noise estimates. CMZ was supported by a CNES fellowship.

\section{References}

Acke, B., van den Ancker, M. E., \& Dullemond, C. P. 2005, A\&A, 436, 209 Bitner, M. A., Richter, M. J., Lacy, J. H., et al. 2007, ApJ, 661, L69 Bouret, J.-C., Martin, C., Deleuil, M., et al. 2003, A\&A, 410, 175 Brittain, S. D., Simon, T., Najita, J. R., \& Rettig, T. W. 2007, ApJ, 659, 685 Carmona, A., van den Ancker, M. E., Henning, T., et al. 2008, A\&A, 477, 839 Cidale, L., Zorec, J., \& Tringaniello, L. 2001, A\&A, 368, 160 Cohen, M., Walker, R. G., \& Witteborn, F. C. 1999, LPI Contributions, 969, 5 
Evans, D. S. 1967, in Determination of Radial Velocities and their Applications, ed. A. H. Batten, \& J. F. Heard, IAU Symp., 57

Finkenzeller, U., \& Jankovics, I. 1984, A\&AS, 57, 285

Fluks, M. A., Plez, B., The, P. S., et al. 1994, A\&AS, 105, 311

Grady, C. A., Bjorkman, K. S., Shepherd, D., et al. 1993, ApJ, 415, L39

Hillenbrand, L. A., Strom, S. E., Vrba, F. J., \& Keene, J. 1992, ApJ, 397, 613

Kraus, S., Preibisch, T., \& Ohnaka, K. 2007, ArXiv e-prints, 711

Lagage, P. O., Pel, J. W., Authier, M., et al. 2004, The Messenger, 117, 12

Lahuis, F., van Dishoeck, E. F., Blake, G. A., et al. 2007, ApJ, 665, 492

Le Petit, F., Nehmé, C., Le Bourlot, J., \& Roueff, E. 2006, ApJS, 164, 506

Leinert, C., Haas, M., Ábrahám, P., \& Richichi, A. 2001, A\&A, 375, 927

Martin, C., Bouret, J.-C., Deleuil, M., et al. 2004, A\&A, 416, L5

Martin-Zaïdi, C., Lagage, P.-O., Pantin, E., \& Habart, E. 2007, ApJ, 666, L117

Martin-Zaïdi, C., Deleuil, M., Le Bourlot, J., et al. 2008, A\&A, 484, 225

Meeus, G., Waters, L. B. F. M., Bouwman, J., et al. 2001, A\&A, 365, 476

Muratorio, G., Rossi, C., \& Friedjung, M. 2006, A\&A, 450, 593

Natta, A., Grinin, V., \& Mannings, V. 2000, Protostars and Planets IV, 559
Polomski, E. F., Telesco, C. M., Piña, R., \& Schulz, B. 2002, AJ, 124, 2207 Richter, M. J., Jaffe, D. T., Blake, G. A., \& Lacy, J. H. 2002, ApJ, 572, L161 Sako, S., Yamashita, T., Kataza, H., et al. 2005, ApJ, 620, 347

Schulte-Ladbeck, R., Shepherd, D., Nordsieck, K., et al. 1992, ApJ, 401, L105 Swings, J. P., \& Allen, D. A. 1971, ApJ, 167, L41

Takahashi, J. 2001, ApJ, 561, 254

Thi, W. F., van Dishoeck, E. F., Blake, G. A., et al. 2001, ApJ, 561, 1074 Valenti, J. A., Johns-Krull, C. M., \& Linsky, J. L. 2000, ApJS, 129, 399 van Boekel, R., Waters, L. B. F. M., Dominik, C., et al. 2003, A\&A, 400, L21 van den Ancker, M., de Winter, D., \& Tjin A Djie, H. 1998, A\&A, 330, 145 van Dishoeck 1992, in Infrared Astronomy with ISO, ed. Th., Encrenaz, \& M. F. Kessler (Nova Science Publisher), 283

Vink, J. S., Drew, J. E., Harries, T. J., et al. 2002, MNRAS, 337, 356

Vinković, D., Ivezić, Ž., Jurkić, T., \& Elitzur, M. 2006, ApJ, 636, 348

Zorec, J., Moujtahid, A., Ballereau, D., \& Chauville, J. 1998, in B[e] stars, ed. A. M. Hubert, \& C. Jaschek, Ap\&SS Library, 233, 55 